\title{
Leishmania infantum-specific production of IFN- $\gamma$ and IL-10 in stimulated blood from dogs with clinical leishmaniosis
}

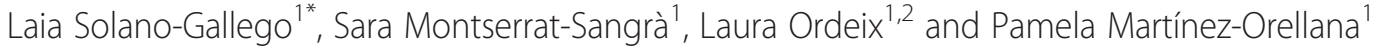

\begin{abstract}
Background: There is limited information available on cytokine profiles in dogs with different degrees of disease severity due to natural infection of Leishmania infantum. The aim of this study was to investigate L. infantum-specific IFN- $\gamma$ and IL-10 production in blood from dogs with leishmaniosis at diagnosis and correlate these findings with disease severity, humoral immune response and blood parasitemia.

Methods: Sixty dogs were diagnosed based on physical examination, routine laboratory tests, L. infantum-specific antibody levels measured by quantitative ELISA and blood parasitemia by real-time PCR. Heparin whole blood was stimulated with L. infantum soluble antigen (LSA) and concanavalin A (ConA) and incubated for 5 days. IFN- $\gamma$ and IL-10 concentrations were measured in supernatants with sandwich ELISAs.

Results: The majority of dogs ( $n=36$ ) were classified as LeishVet stage II (moderate disease). The rest of the dogs were classified as stage I $(n=10)$, III $(n=10)$ and IV $(n=4)$. Dogs classified with stage I and lla presented significantly higher $(P=0.02)$ LSA IFN- $\gamma$ concentrations, lower $(P<0.0001)$ antibody levels and a tendency for lower blood parasitemia $(P=0.1)$ than dogs classified with stages IIb, III or IV while no differences in ConA IFN- $\gamma$ or IL-10 concentrations were observed among groups. Thirty-five dogs produced significantly higher LSA IFN- $\gamma$ (mean \pm SD: $2320 \pm 3960 \mathrm{pg} / \mathrm{ml}$ ) and ConA IFN- $\gamma$ (mean \pm SD: $7887 \pm 7273 \mathrm{pg} / \mathrm{ml}$ ) when compared with 25 dogs that did not produce detectable LSA IFN- $\gamma$ but produced ConA IFN- $\gamma$ (mean \pm SD: $4917 \pm 5233 \mathrm{pg} / \mathrm{ml}$ ). IFN- $\gamma$ producer dogs presented lower (mean \pm SD: $5750 \pm$ 14,082 ELISA units (EU), $P=0.001$ ) antibody levels and blood parasitemia (mean \pm SD: $5 \pm 10$ parasites $/ \mathrm{ml}, P=0.001$ ) when compared with IFN- $\gamma$ non-producers (mean \pm SD: 19,638 $\pm 28,596$ EU and $1100 \pm 5112$ parasites $/ \mathrm{ml}$ ), respectively. LSA IL-10 was not detectable in 34 dogs while 49 dogs secreted ConA IL-10 (mean \pm SD of $90 \pm 103$ pg/ml). LSA IFN- $\gamma$ concentration was negatively correlated with blood parasitemia and antibody levels and positively correlated with ConA IFN- $\gamma$ and LSA IL-10 concentrations.
\end{abstract}

Conclusions: The results of this study demonstrate that sick dogs lacking L. infantum specific IFN- $\gamma$ production in stimulated whole blood produce a strong humoral response, have a high blood parasitemia and severe clinical disease. IL-10 does not appear to be a marker of disease severity.

Keywords: Leishmania infantum, Dog, IFN-ץ, IL-10, Disease, Clinical staging

\footnotetext{
* Correspondence: laia.solano@uab.cat

'Departament de Medicina i Cirurgia Animals, Facultat de Veterinària,

Universitat Autònoma de Barcelona, Bellaterra, Spain

Full list of author information is available at the end of the article
} 


\section{Background}

Species of Leishmania are obligate intracellular protozoan parasites that are transmitted by female phlebotomine sandflies. Canine leishmaniosis is a zoonotic disease caused by several species of Leishmania. Although other Leishmania species have been reported to infect dogs such as L. major [1], L. tropica and L. braziliensis [2-4], Leishmania infantum is the most common species in the Mediterranean basin [5, 6]. Dogs are considered the principal reservoir for leishmaniosis in people due to $L$. infantum in the Mediterranean basin, Middle East and South America [5].

Both innate and adaptive immune responses play a role in the outcome of Leishmania infection. Classically, the major role against the parasite is played by the adaptive immune response, characterized by the balance between T-helper 1 (Th1) and T-helper 2 (Th2) response. Th1 secrete interleukin-2 (IL-2), TNF-alpha and interferongamma (IFN- $\gamma$ ) and this response is associated with controlling infection while Th2 cells secrete interleukin-4 (IL-4), interleukin-5 (IL-5), interleukin-10 (IL-10), and transformation growth factor beta (TGF- $\beta$ ) and correlate with disease progression $[7,8]$.

Dogs show a broad range of clinical manifestations and immune responses [9] in canine leishmaniosis. Canine L. infantum infection can manifest as a chronic subclinical infection, self-limiting disease, or non-selflimiting illness $[5,6]$ as in humans [10]. In addition, several degrees of disease severity are found in dogs ranging from mild disease to severe fatal disease with different clinical outcomes, prognosis and treatment options. Therefore, a clinical staging system is currently used in the clinical setting [6]. The two extremes of this clinical spectrum are characterized by: (1) "Resistant" healthy dogs with a protective $\mathrm{CD} 4^{+} \mathrm{T}$-cell-mediated immune response characterized by production of Th1 cytokineslike such as IFN- $\gamma$, IL-2 and TNF- $\alpha$, which induce anti-Leishmania activity by apoptosis of parasites in macrophages via nitric oxide (NO) metabolism [11], and (2) sick dogs which are characterized by a marked humoral immune response, absent or reduced cell mediated immunity with high levels of IL- 10 and TGF- $\beta$ and high parasite burden, which is detrimental to the animal $[5,12]$.

Unfortunately, there are few and poorly standardized assays to evaluate T-cell mediated immunity responses [13] such as leishmanin skin test [14, 15] and lymphocyte proliferation $[16,17]$ in the dog. The results of cytokine profiles in canines with $L$. infantum infection are frequently limited and fragmentary [9]. Cytokines including IFN- $\gamma$ and IL-10 have been studied in several organs with different techniques in dogs with poorly defined clinical classification and different states of infection [5, 9]. In addition, cytokines have been mainly investigated in vaccinated [18] or in experimentally- infected dogs [17] but limited information is available in sick dogs with different degrees of disease severity due to $L$. infantum natural infection $[17,19]$. The aim of this study was to investigate $L$. infantum-specific IFN- $\gamma$ and IL-10 production in stimulated blood in dogs with clinical leishmaniosis at the time of diagnosis and correlate these with disease severity, the humoral immune response and blood parasitemia.

\section{Methods \\ Dogs}

Sixty dogs with clinical leishmaniosis were prospectively enrolled from January 2014 to February 2016 at the time of diagnosis. The dogs were from different Catalonian and Balearic veterinary centers from Spain: Fundació Hospital Clínic Veterinari (Bellaterra, Barcelona), Hospital Ars Veterinaria (Barcelona), Hospital Mediterrani Veterinaris (Reus, Tarragona), Consultori Montsant (Falset, Tarragona) and Hospital Mon Veterinari (Manacor, Mallorca). The diagnosis of canine leishmaniosis was made based on the results of the physical examination, a complete blood count (CBC) using Siemens Advia 120 Haematology System (Siemens Healthcare $\mathrm{GmbH}$, Germany), a biochemical profile including creatinine, urea, total proteins, ALT and total cholesterol by Olympus AU400 Chemistry Analyzer (CLIAwaived, USA), protein serum electrophoresis by Hydrasys ${ }^{\circledR}$ (Sebia Electrophoresis, USA), urinalysis with urinary protein/creatinine ratio (UPC) and quantitative serology for the detection of $L$. infantum specific antibodies by means of a serial dilution in house ELISA [20-22]. Cytological evaluation of any lesion or cutaneous histology and/or immunohistochemistry for Leishmania was also performed in some cases as described elsewhere when needed [23]. In addition, DNA was extracted from blood samples and L. infantum realtime PCR (RT-PCR) was performed with an absolute quantification as previously described [22, 24]. Dogs were classified in four clinical stages (stage I-mild disease, IImoderate disease including substages IIa and IIb, IIIsevere disease and IV-very severe disease) at the time of diagnosis as previously described [6].

\section{Whole blood assay}

The leucocyte concentration including lymphocyte and neutrophil concentrations was very similar between dogs measured by a CBC. Heparinized blood was diluted to a ratio of 1:10 with Rosewell Park Memorial Institute (RPMI) 1640 medium with stable glutamine and $25 \mathrm{mM}$ hepes (Biowest ${ }^{\circ}, \mathrm{USA}$ ) supplemented with $60 \mu \mathrm{g} / \mathrm{ml}$ of penicillin, $100 \mu \mathrm{g} / \mathrm{ml}$ streptomycin (Life Technologies ${ }^{\mathrm{TM}}$, USA) and 10 \% Fetal Bovine Serum Premium South America Origin (Biowest $\left.{ }^{\oplus}, \mathrm{USA}\right)$. Five hundred $\mu \mathrm{l}$ of heparinized blood was mixed with $4.5 \mathrm{ml}$ of complete medium as described above per each well and incubated in 12-well flat bottom 
plastic culture plates 3596 (Costar Corning, NY, USA). Three different treatment conditions were established: (i) medium alone; (ii) medium with $L$. infantum soluble antigen (LSA) at a concentration of $10 \mu \mathrm{g} / \mathrm{ml}$ provided by Dr. Cristina Riera (Facultat de Farmacia, Universitat de Barcelona); and (iii) medium with mitogen concanavalin A (ConA) (100 mg Medicago $^{\circ}$, Sweden) at a concentration of $10 \mu \mathrm{g} / \mathrm{ml}$. The plates were incubated for 5 days at $37{ }^{\circ} \mathrm{C}$ in $5 \%$ of $\mathrm{CO}_{2}$ air. Following incubation, blood was centrifuged at $300 \mathrm{~g}$ for 10 minutes and the supernatant was collected and stored at $-80{ }^{\circ} \mathrm{C}$ until used.

Sandwich ELISAs for the determination of IFN- $\gamma$ and IL-10 Cytokine analysis of IFN- $\gamma(n=60)$ and IL-10 $(n=53)$ were performed according to the manufacturer's instructions (DuoSet ${ }^{\circ}$ ELISA by Development System $R \& D^{T M}$, UK) using 96 well cell flat bottom plates (Costar ${ }^{\circledR}$ Corning, USA). Slight modifications were done for the IFN- $\gamma$ and IL-10 ELISAs. The standard curve for IFN- $\gamma$ started with $8000 \mathrm{pg} / \mathrm{ml}$ and two-fold dilutions were made until $62.5 \mathrm{pg} / \mathrm{ml}$ concentration. Standard curve for IL-10 started with $2000 \mathrm{pg} / \mathrm{ml}$ and two-fold dilutions were made until $15.6 \mathrm{pg} / \mathrm{ml}$ concentration was obtained. Supernatants treated with ConA were diluted 1:1 with reagent diluent. Duplicates of all supernatants studied were performed in all ELISAs. Optical density was measured with an ELISA reader (Anthos 2020) at wavelength of $450 \mathrm{~nm}$. The standard curve for each cytokine was calculated using a computer generated four parameter logistic curve-fit with program MyAssays (http://www.myassays.com/). Plates were repeated when $R^{2}$-value of standard curve was below 0.98 . Dogs were classified as IFN- $\gamma$ producers when $L$. infantum specific IFN- $\gamma$ concentration was detectable after subtracting medium alone. Dogs were classified as IFN- $\gamma$ non-producers when $L$. infantum specific IFN- $\gamma$ concentration subtracting medium alone was at not detectable levels. IL-10 detectable concentrations were considered after subtracting medium alone for LSA and ConA stimulations.

\section{Statistical analysis}

The statistical analysis was performed using the SPSS 22.0 for Windows software (SPSS Inc., USA). A nonparametric Mann-Whitney U-test was used to compare groups. A non-parametric Wilcoxon signed-rank test was used to compare paired continuous variables. The Spearman's correlation was used to evaluate differences in cytokine production, the level of antibodies and blood parasitemia of the dogs studied. Differences were considered significant with a $5 \%$ significance level $(P<0.05)$.

\section{Results}

Description of clinical data of dogs, clinical staging and specific $L$. infantum IFN- $\gamma$ response classifications

Both sexes were represented by 25 females and 35 males. The median of age was four years with a range from five months to 12 years. Forty-four purebred dogs belonging to more than 25 breeds and 16 mixed breed dogs were included.

Based on clinicopathological findings and serological tests, the majority of dogs $(n=36)$ were classified as LeishVet stage II (moderate disease) and this stage was further divided into stage IIa $(n=27)$ and stage IIb $(n=9)$. The rest of the dogs were classified as stage I $(n=10)$, stage III $(n=10)$ and stage IV $(n=4)$. All dogs in stage I were diagnosed with papular dermatitis due to L. infantum and presented negative to low positive antibody levels. The rest of dogs presented typical clinicopathological findings and mainly medium to high positive antibody levels. The results of signalment, serology, clinical signs and laboratory abnormalities observed in dogs with clinical leishmaniosis classified in four clinical stages are shown in Table 1.

Thirty-five dogs $(58 \%)$ were classified as IFN- $\gamma$ producers. The clinical staging of these dogs was: nine dogs had stage I (25.7 \%), 17 dogs stage IIa (48.5\%), three dogs stage IIb (8.5\%), five dogs stage III (14.2\%) and one dog stage IV (2.8 \%) (Fig. 1a). In contrast, 25 dogs did not secrete IFN- $\gamma$ after LSA stimulation. All 25 dogs (42\%) were classified as IFN- $\gamma$ non-producers. The clinical staging of IFN- $\gamma$ non-producers was as follows: one $\operatorname{dog}(4 \%)$ was classified as stage I; ten dogs as stage IIa (40\%); six dogs as stage IIb (24 \%); five dogs as stage III $(20 \%)$ and three dogs (12\%) as stage IV (Fig. 1b).

\section{Antibody response}

The results of antibody levels based on clinical staging and IFN- $\gamma$ classification are shown in Tables 2 and 3. Dogs classified with stage I and IIa presented significantly lower antibodies (Mann-Whitney U-test: $\mathrm{Z}=-4.37, P<0.0001$ ) (mean \pm SD: $4050 \pm 9947 \mathrm{EU}$ ) than dogs classified with stages IIb, III or IV (mean \pm SD: 23,580 $\pm 30,308 \mathrm{EU})$.

IFN $-\gamma$ producer dogs (mean \pm SD: $5750 \pm 14,082 \mathrm{EU}$ ) showed statistically significant lower levels of antibodies (Mann-Whitney U-test: $Z=-3.23, \quad P=0.001$ ) when compared with IFN $-\gamma$ non-producer dogs (mean \pm SD: 19,638 $\pm 28,596 \mathrm{EU}$ ) (Table 2).

\section{Blood parasite load by real-time PCR}

The results of blood parasitemia based on clinical staging and IFN- $\gamma$ classification are shown in Tables 2 and 3. Blood PCR was positive in 40 dogs $(66.6 \%)$ out of 59 dogs. In addition, dogs classified with stage I and IIa group presented a tendency (Mann-Whitney U-test: $Z=-1.53, P=0.1)$ for lower blood parasitemia $(5.3 \pm 10.2$ 
Table 1 Clinicopathological findings in dogs with clinical leishmaniosis based on LeishVet clinical staging [6]

\begin{tabular}{|c|c|c|c|c|}
\hline Clinical staging & $\begin{array}{l}\text { Gender/median } \\
\text { [range] of age in } \\
\text { months }\end{array}$ & Serology & $\begin{array}{l}\text { Clinical signs (number of dogs/total } \\
\text { number of dogs, \%) }\end{array}$ & $\begin{array}{l}\text { Laboratory findings (number of dogs/ } \\
\text { total number of dogs, \%) }\end{array}$ \\
\hline $\begin{array}{l}\text { I-mild disease } \\
(n=10)\end{array}$ & $\begin{array}{l}5 \text { females and } \\
4 \text { males/15.2 [5-40] }\end{array}$ & $\begin{array}{l}\text { Negative to } \\
\text { low positive } \\
\text { antibody levels }\end{array}$ & Papular dermatitis (10/10, $100 \%)$ & No abnormalities observed \\
\hline $\begin{array}{l}\text { lla-moderate } \\
\text { disease }(n=27)\end{array}$ & $\begin{array}{l}11 \text { females and } \\
16 \text { males/51 [5-153] }\end{array}$ & $\begin{array}{l}\text { Low to high positive } \\
\text { antibody levels }\end{array}$ & $\begin{array}{l}\text { Cutaneous lesions }(20 / 27,74 \%) \text { : } \\
\text { - Exfoliative dermatitis }(9 / 27,33 \%) \\
\text { - Ulcerative dermatitis }(9 / 27,33 \%) \\
\text { - Alopecia (7/27, } 26 \%) \\
\text { - Nodular dermatitis }(3 / 27,11 \%) \\
\text { - Papular dermatitis }(2 / 27,7 \%) \\
\text { - Ischemic dermatopathy }(1 / 27,4 \%) \\
\text { Lymphadenomegaly }(19 / 27,70 \%) \\
\text { Weight loss }(8 / 27,30 \%) \\
\text { Fever }(5 / 27,19 \%) \\
\text { Ocular lesions }(4 / 27,15 \%) \\
\text { - Conjunctivitis }(3 / 27,11 \%) \\
\text { - Blepharitis }(1 / 27,4 \%) \\
\text { Lameness }(4 / 27,15 \%) \\
\text { Vomiting and/or diarrhea }(2 / 27,7 \%) \\
\text { Inspiratory dyspnea }(1 / 27,4 \%) \\
\text { Masseter myositis }(1 / 27,4 \%) \\
\text { Muscle atrophy }(1 / 27,4 \%) \\
\text { Epistaxis (1/27, } 4 \%)\end{array}$ & $\begin{array}{l}\text { Mild to moderate normocytic normochromic } \\
\text { non-regenerative anemia }(8 / 26,31 \% ; 3 / 26, \\
12 \% \text {, respectively) } \\
\text { Mild leukocytosis with mature neutrophilia } \\
\text { (2/26, } 8 \%) \\
\text { Mild to moderate thrombocytopenia }(2 / 26,8 \%) \\
\text { Mild leucopenia with lymphopenia and } \\
\text { neutropenia }(2 / 26,8 \%) \\
\text { Hyperproteinemia }(13 / 27,48 \%) \\
\text { Hypergammaglobulinemia }(14 / 27,52 \%) \\
\text { Hyperbetaglobulinemia }(15 / 27,55 \%) \\
\text { Hypoalbuminemia }(6 / 26,23 \%)\end{array}$ \\
\hline $\begin{array}{l}\text { llb-moderate } \\
\text { disease }(n=9)\end{array}$ & $\begin{array}{l}2 \text { females and } 9 \\
\text { males/43 [9-147] }\end{array}$ & $\begin{array}{l}\text { Medium to high } \\
\text { positive antibody } \\
\text { levels }\end{array}$ & $\begin{array}{l}\text { Cutaneous lesions }(7 / 9,77 \%) \text { : } \\
\text { - Exfoliative dermatitis (3/9, } 33 \%) \\
\text { - Ulcerative dermatitis (3/9, } 33 \%) \\
\text { - Alopecia }(2 / 9,22 \%) \\
\text { Lymphadenomegaly }(6 / 9,67 \%) \\
\text { Weight loss }(5 / 9,56 \%) \\
\text { Ocular lesions }(4 / 9,44 \%) \\
\text { - Conjunctivitis }(2 / 9,22 \%) \\
\text { - Bilateral blepharitis }(2 / 9,22 \%) \\
\text { Vomiting and/or diarrhea (3/9, } 33 \%) \\
\text { Lethargy (2/9, } 22 \%) \\
\text { Lameness and articular pain (1/9, } \\
33 \%) \\
\text { Polyuria and polydipsia }(1 / 9,11 \%)\end{array}$ & $\begin{array}{l}\text { Mild to moderate normocytic normochromic } \\
\text { non-regenerative anemia }(6 / 9,67 \% ; 2 / 9,22 \% \text {, } \\
\text { respectively) } \\
\text { Mild leukocytosis with mild mature neutrophilia } \\
\text { (2/9, } 22 \%) \\
\text { Lymphopenia }(3 / 9,33 \%) \\
\text { Hyperproteinemia }(7 / 9,77 \%) \\
\text { Hypergammaglobulinemia }(9 / 9,100 \%) \\
\text { Hyperbetaglobulinemia }(6 / 9,66 \%) \\
\text { Hypoalbuminemia }(6 / 9,66 \%)\end{array}$ \\
\hline
\end{tabular}

III-severe $\quad 5$ females and $4 \quad$ Medium to high disease $(n=10)$ males/85 [37-132] positive antibody levels
Lymphadenomegaly (7/10, $70 \%)$

Cutaneous lesions $(6 / 10,60 \%)$ :

- Exfoliative dermatitis (4/10, $40 \%)$

- Alopecia $(1 / 10,10 \%)$

- Ulcerative dermatitis (2/10, $20 \%)$

Weight loss $(5 / 10,50 \%)$

Ocular lesions (3/10, $30 \%)$ :

- Bilateral blepharitis (1/10, $10 \%)$

- Conjunctivitis (1/10, $10 \%)$

- Superficial keratitis $(1 / 10,10 \%)$

Lethargy and anorexia (2/10, $20 \%)$

Vomiting and/or diarrhea (2/10,

$20 \%)$

Splenomegaly (2/10, $20 \%)$

Lameness and articular pain (1/10,

$10 \%)$

Muscle atrophy $(1 / 10,10 \%)$

Epistaxis (1/10, $11 \%)$

Polyuria and polydipsia (1/10, $10 \%)$

Fever $(1 / 10,10 \%)$

Chronic weakness (1/10, $10 \%)$
Mild normocytic normochromic non-regenerative anemia $(6 / 9,67 \%)$

Moderate regenerative anemia (2/9, $22 \%)$

Thrombocytosis $(1 / 9,11 \%)$

Mild thrombocytopenia $(1 / 9,11 \%)$

Mild leukocytosis with mature neutrophilia

$(2 / 9,22 \%)$

Mild leukopenia with lymphopenia and

neutropenia $(1 / 9,11 \%)$

Lymphopenia $(1 / 9,11 \%)$

Mild lymphocytosis $(1 / 9,11 \%)$

Hyperproteinemia $(8 / 10,80 \%)$

Hypergammaglobulinemia $(9 / 10,90 \%)$

Hyperbetaglobulinemia $(9 / 10,90 \%)$

Hypoalbuminemia $(9 / 10,90 \%)$

Proteinuria (UPC) (10/10, $100 \%)$ 
Table 1 Clinicopathological findings in dogs with clinical leishmaniosis based on LeishVet clinical staging [6] (Continued)

\begin{tabular}{|c|c|c|c|c|}
\hline Clinical staging & $\begin{array}{l}\text { Gender/median } \\
\text { [range] of age in } \\
\text { months }\end{array}$ & Serology & $\begin{array}{l}\text { Clinical signs (number of dogs/total } \\
\text { number of dogs, \%) }\end{array}$ & $\begin{array}{l}\text { Laboratory findings (number of dogs/ } \\
\text { total number of dogs, \%) }\end{array}$ \\
\hline $\begin{array}{l}\text { IV-very severe } \\
\text { disease }(n=4)\end{array}$ & $\begin{array}{l}2 \text { females and } 2 \\
\text { males/7.5 [23-120] }\end{array}$ & $\begin{array}{l}\text { Medium to high } \\
\text { positive antibody } \\
\text { levels }\end{array}$ & $\begin{array}{l}\text { Weight loss ( } 3 / 4,75 \%) \\
\text { Cutaneous lesions }(3 / 4,75 \%): \\
\text { - Exfoliative dermatitis }(2 / 4,50 \%) \\
\text { - Ulcerative dermatitis }(1 / 4,25 \%) \\
\text { - Onicogriphosis }(1 / 4,25 \%) \\
\text { - Ulcerative and nodular blepharitis } \\
\text { (1/4, } 25 \%) \\
\text { Vomiting and/or diarrhea }(2 / 4,50 \%) \\
\text { Lymphadenomegaly }(1 / 4,25 \%) \\
\text { Cachexy }(1 / 4,25 \%) \\
\text { Hypertension }(1 / 4,25 \%) \\
\text { Cardiac insufficiency }(1 / 4,25 \%) \\
\text { Keratoconjunctivitis }(1 / 4,25 \%) \\
\text { Chronic weakness }(1 / 4,25 \%) \\
\text { Paraparesis }(1 / 4,25 \%)\end{array}$ & $\begin{array}{l}\text { Mild normocytic normochromic non } \\
\text { regenerative anemia }(1 / 4,25 \% ; 3 / 4,75 \% \text {, } \\
\text { respectively) } \\
\text { Thrombocytosis }(2 / 4,50 \%) \\
\text { Lymphopenia }(1 / 4,25 \%) \\
\text { Mild leukocytosis with mature neutrophilia } \\
(1 / 4,25 \%) \\
\text { Total protein }(4 / 4,100 \%) \\
\text { Hypoalbuminemia }((4 / 4,100 \%) \\
\text { Hypergammaglobulinemia }(3 / 3,100 \%) \\
\text { Hyperbetaglobulinemia }(3 / 3,100 \%) \\
\text { Proteinuria (UPC) }(4 / 4,100 \%) \\
\text { Renal azotemia }(3 / 3,100 \%)\end{array}$ \\
\hline
\end{tabular}

UPC: urinary protein creatinine ratio

parasites $/ \mathrm{ml}$ ) than dogs classified with stages IIb, III or IV group (1255 \pm 5464 parasites $/ \mathrm{ml})$.

Interestingly, 14 IFN- $\gamma$ producer dogs and five IFN- $\gamma$ non-producers were negative by $\mathrm{PCR}$ at the time of diagnosis. Twenty-one (60\%) out of 35 IFN- $\gamma$ producer dogs were positive by PCR at the time of diagnosis. Nineteen (76\%) out of 25 IFN- $\gamma$ non-producer dogs were positive to PCR at the time of diagnosis. IFN- $\gamma$ producer dogs presented a statistically significant lower parasitemia (Mann-Whitney U-test: $Z=-3.22, P=0.001$ ) when compared with IFN- $\gamma$ non-producers (Table 3).

\section{IFN- $\gamma$}

The results of ConA and L. infantum specific IFN- $\gamma$ concentrations based on clinical staging and IFN- $\gamma$ classification are shown in Tables 2 and 3. IFN- $\gamma$ concentrations in supernatants of blood stimulated with LSA and ConA from all dogs studied were (mean \pm SD) $1354 \pm 3220 \mathrm{pg} /$ $\mathrm{ml}$ and $6649 \pm 6618 \mathrm{pg} / \mathrm{ml}$, respectively. A significantly higher concentration of IFN- $\gamma$ on ConA stimulated blood was found when compared with LSA stimulated blood (Wilcoxon signed-rank test: $Z=-6.15, \quad P<0.0001$ ). Dogs classified with stage I and IIa group presented significantly higher (Mann-Whitney U-test: $Z=-2.32, P=$ 0.02) LSA IFN- $\gamma$ concentrations (mean \pm SD: $1939 \pm 3952$ pg/ $\mathrm{ml}$ ) than dogs classified with stages IIb, III or IV group (mean \pm SD: $411 \pm 862 \mathrm{pg} / \mathrm{ml}$ ) while no differences in ConA IFN- $\gamma$ concentrations were observed among these two groups.

IFN- $\gamma$ producer dogs $(n=35)$ showed a significantly higher (Mann-Whitney U-test: $Z=-6.78, P<0.0001)$ LSA IFN $-\gamma$ concentration (mean \pm SD of $2320 \pm 3961 \mathrm{pg} / \mathrm{ml}$ ) when compared with IFN- $\gamma$ non-producers $(n=25)$ (mean $\pm \mathrm{SD}$ of $0 \pm 0 \mathrm{pg} / \mathrm{ml}$ ). The mean $\pm \mathrm{SD}$ of ConA IFN- $\gamma$ concentration from IFN- $\gamma$ producer dogs was $7887 \pm 7273 \mathrm{pg} / \mathrm{ml}$ while the mean $\pm \mathrm{SD}$ of ConA IFN- $\gamma$ concentration from IFN- $\gamma$ non-producers was $4917 \pm 5233 \mathrm{pg} / \mathrm{ml}$. Statistical differences were found between IFN- $\gamma$ producers and non-producers for IFN$\gamma$ concentration after stimulation with ConA (MannWhitney U-test: $Z=-2.04, P=0.041)$.
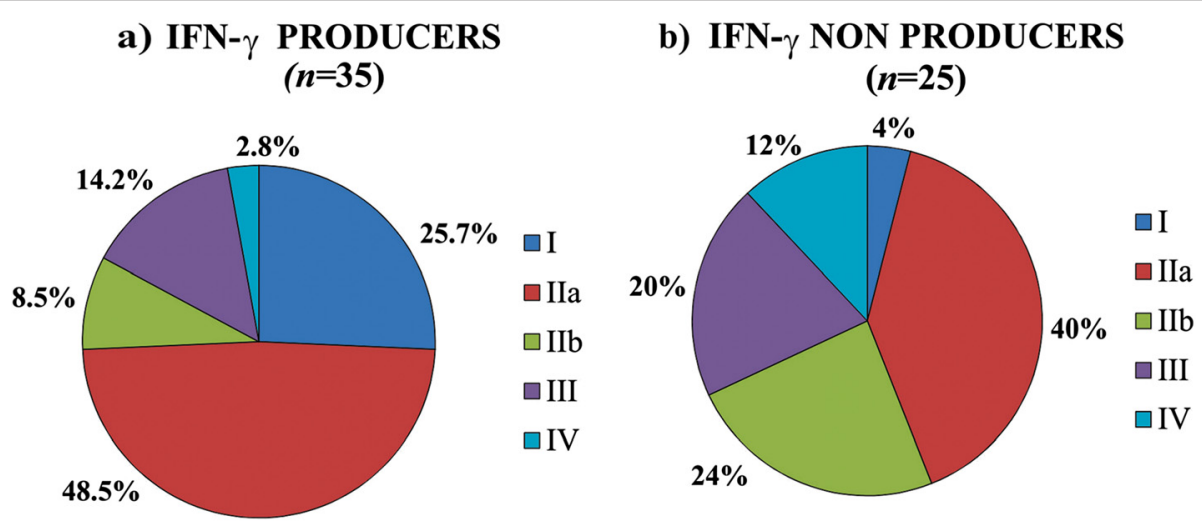

Fig. 1 Percentages of sick dogs according with IFN- $\gamma$ production after LSA stimulation and clinical staging. a IFN- $\gamma$ producers $(n=35)$ and $\mathbf{b}$ IFN- $\gamma$ non-producers $(n=25)$ 
Table 2 Summary of parameters studied based on clinical staging distribution of sick dogs

\begin{tabular}{|c|c|c|c|c|c|c|c|}
\hline \multirow{2}{*}{$\begin{array}{l}\text { Clinical } \\
\text { staging }\end{array}$} & \multicolumn{7}{|c|}{ Mean \pm standard deviation } \\
\hline & Number of dogs & IFN-ץ LSA (pg/ml) & IFN- $\gamma$ ConA (pg/ml) & IL-10 LSA (pg/ml) & IL-10 ConA (pg/ml) & PCR (parasite/ml) & ELISA (EU) \\
\hline I & 10 & $2022 \pm 2687^{h}$ & $4029 \pm 3942^{i}$ & $17 \pm 18$ & $82 \pm 46$ & $3.7 \pm 10.4^{d}$ & $46 \pm 51^{a}$ \\
\hline$\| a$ & 27 & $1909 \pm 4373$ & $7943 \pm 7115^{j}$ & $8 \pm 14$ & $57 \pm 50$ & $6 \pm 10^{e}$ & $5533 \pm 11338^{b}$ \\
\hline llb & 9 & $539 \pm 1231$ & $6777 \pm 6068$ & $7 \pm 14$ & $144 \pm 130$ & $101 \pm 285^{f}$ & $23,287 \pm 27636$ \\
\hline III & 10 & $423 \pm 629$ & $8004 \pm 7914^{k}$ & $15 \pm 19$ & $94 \pm 167$ & $20 \pm 26^{9}$ & $12,416 \pm 20,227^{c}$ \\
\hline IV & 4 & $94 \pm 187$ & $792 \pm 350$ & $5 \pm 9$ & $77 \pm 112$ & $6337 \pm 12501$ & $52,151 \pm 44,365$ \\
\hline Total & 60 & $1354 \pm 3220$ & $6649 \pm 6618$ & $10 \pm 15$ & $83 \pm 102$ & $458 \pm 3293$ & $11,537 \pm 22,240$ \\
\hline
\end{tabular}

Parameters under study were evaluated in relationship to clinical staging and letters from ${ }^{\mathrm{a}}$ to ${ }^{\mathrm{b}}$ correspond to statistically significant findings

Results for ELISA units ${ }^{\mathrm{a}} \mathrm{I}<\mathrm{Ila}$, Ilb, III, IV (Mann-Whitney U-test: $\left.Z=-3.7, P<0.0001\right)$; ${ }^{\mathrm{b}} \mathrm{lla}>\mathrm{I}$ (Mann-Whitney U-test: $\left.Z=-4.4, P<0.0001\right)$ and Ila $<$ IIb, IV (MannWhitney U-test: $Z=-2.7, P=0.002)$; ${ }^{C}$ III $<$ IV (Mann-Whitney U-test: $\left.Z=-2.1, P=0.036\right)$

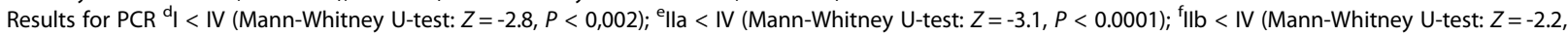

$P=0.028) ;{ }^{9}$ III $<$ IV (Mann-Whitney U-test: $\left.Z=-2.2, P=0.024\right)$

Results for IFN- $\gamma$ LSA $\mathrm{h}$ I Ilb, III, IV (Mann-Whitney U-test: $Z=-2.1, P<0.035$ )

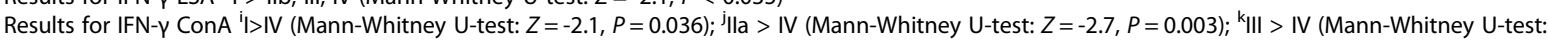

$Z=-2.5, P=0.008)$

Abbreviations: LSA L. infantum soluble antigen, ConA concavalin A

\section{IL-10}

The results of ConA and L. infantum specific IL-10 concentrations based on clinical staging and IFN- $\gamma$ classification are shown in Tables 2 and 3. The mean \pm SD of IL-10 concentrations in supernatants of blood stimulated with LSA and ConA from all dogs studied was $10 \pm 15 \mathrm{pg} / \mathrm{ml}$ and $83 \pm 102 \mathrm{pg} / \mathrm{ml}$, respectively. A significantly higher concentration of IL-10 in ConA stimulated blood was found when compared with LSA stimulated blood (Wilcoxon signed-rank test: $Z=-5.62, P<0.0001$ ). Moreover, 34 out of $53 \operatorname{dogs}(64 \%)$ did not produce IL-10 after LSA stimulation $(0 \mathrm{pg} / \mathrm{ml})$ while the rest of the dogs $(n=19 ; 36 \%)$ produced significantly higher concentrations (Mann-Whitney U-test: $Z=-6.46, P<0.0001$ ) of IL-10 (mean \pm SD of $27.1 \pm 13.3 \mathrm{pg} / \mathrm{ml}$ ). Only four dogs did not produce IL-10 after ConA stimulation. Statistical analysis showed significantly higher concentrations of IL-10 in IFN- $\gamma$ producer dogs after LSA stimulation when compared with IFN- $\gamma$ non-producers (Mann-Whitney U- test: $Z=-3.60, P<0.0001)$. No significant differences in IL-10 concentrations were observed after ConA stimulation between IFN- $\gamma$ producers and IFN- $\gamma$ non-producers.

Concentration of IL-10 from dogs classified with stages I and IIa group $(10 \pm 15 \mathrm{pg} / \mathrm{ml})$ did not present significant differences when compared with dogs classified with stages IIb, III and IV group $(10 \pm 16 \mathrm{pg} / \mathrm{ml})$. No differences in ConA IL-10 concentrations were observed between stage I and IIa group and stages IIb, III and IV group.

\section{Correlation between parameters studied}

The correlations among parameters studied are listed in Table 4.

IFN- $\gamma$ concentration after LSA stimulation was significantly negatively correlated with blood PCR and antibody levels as well as positively correlated with IFN- $\gamma$ concentration after ConA stimulation and IL-10 concentration after LSA stimulation. No further significant correlations were found with IL-10 production.

Table 3 Summary of parameters based on IFN- $\gamma$ producer versus IFN- $\gamma$ non-producer dogs

\begin{tabular}{|c|c|c|c|c|c|c|c|c|c|c|}
\hline \multirow{2}{*}{$\begin{array}{l}\text { Clinical } \\
\text { staging }\end{array}$} & \multicolumn{5}{|c|}{ IFN- $\gamma$ producers (mean \pm standard deviation) } & \multicolumn{5}{|c|}{ IFN- $\gamma$ non-producers (mean \pm standard deviation) } \\
\hline & $\begin{array}{l}\text { Number } \\
\text { of dogs }\end{array}$ & $\begin{array}{l}\text { IFN-y LSA } \\
(\mathrm{pg} / \mathrm{ml})\end{array}$ & $\begin{array}{l}\text { IL-10 LSA } \\
(\mathrm{pg} / \mathrm{ml})\end{array}$ & $\begin{array}{l}\text { PCR } \\
\text { (parasite/ml) }\end{array}$ & ELISA (EU) & $\begin{array}{l}\text { Number } \\
\text { of dogs }\end{array}$ & $\begin{array}{l}\text { IFN-y LSA } \\
(\mathrm{pg} / \mathrm{ml})\end{array}$ & $\begin{array}{l}\text { IL-10 LSA } \\
(\mathrm{pg} / \mathrm{ml})\end{array}$ & $\begin{array}{l}\text { PCR } \\
\text { (parasite/ml) }\end{array}$ & ELISA (EU) \\
\hline । & 9 & $2247 \pm 2749$ & $18 \pm 20$ & $4.1 \pm 11$ & $50 \pm 53$ & 1 & $0 \pm 0$ & $15 \pm 0$ & $0 \pm 0$ & $13 \pm 0$ \\
\hline Ila & 17 & $3031 \pm 5240$ & $12 \pm 16$ & $2 \pm 4$ & $2660 \pm 7140$ & 10 & $0 \pm 0$ & $3 \pm 8$ & $12 \pm 14$ & $10,418 \pm 15,420$ \\
\hline$\| \mathrm{b}$ & 3 & $1616 \pm 1860$ & $14 \pm 23$ & $0.1 \pm 0.1$ & $14,373 \pm 15,473$ & 6 & $0 \pm 0$ & $3.3 \pm 8$ & $162 \pm 360$ & $27,744 \pm 32,476$ \\
\hline III & 5 & $846 \pm 665$ & $29 \pm 18$ & $12 \pm 19$ & $7755 \pm 5590$ & 5 & $0 \pm 0$ & $0 \pm 0$ & $29 \pm 31$ & $17,077 \pm 28,897$ \\
\hline IV & 1 & $375 \pm 0$ & $19 \pm 0$ & $35 \pm 0$ & $73,701 \pm 0$ & 3 & $0 \pm 0$ & $0 \pm 0$ & $8438 \pm 14,419$ & $44,967 \pm 51,408$ \\
\hline Total & 35 & $2320 \pm 3961^{a}$ & $16 \pm 18^{\mathrm{b}}$ & $5 \pm 10$ & $5750 \pm 14,082$ & 25 & $0 \pm 0$ & $2.4 \pm 7$ & $1100 \pm 5112^{c}$ & $19,638 \pm 28,596^{d}$ \\
\hline
\end{tabular}

Statistical study was performed to compare IFN- $\gamma$ producers versus non-IFN- $\gamma$ producers. Letters a to $d$ correspond to statistically significant differences

Results for IFN- $\gamma$ LSA, IFN- $\gamma^{\mathrm{a}}$ producers $>$ non-IFN- $\gamma$ producers (Mann-Whitney U-test: $Z=-6.7, P<0.0001$ )

Results for IL-10 LSA, IFN- $\gamma^{\mathrm{b}}$ producers $>$ non-IFN- $\gamma$ producers (Mann-Whitney U-test: $Z=-3.60, P<0.0001$ )

Results for PCR, IFN- $\gamma^{c}$ producers $<$ non-IFN- $\gamma$ producers (Mann-Whitney U-test: $Z=-3.2, P=0.001$ )

Results for ELISA units, IFN- $\gamma^{d}$ producers $<$ non-IFN- $\gamma$ producers (Mann-Whitney U-test: $Z=-3.2, P=0.001$ )

Abbreviations: LSA L. infantum soluble antigen 
Table 4 Correlation between IFN- $\gamma$ concentration after LSA stimulation and the level of antibodies, blood parasitemia and cytokine production

\begin{tabular}{lcc}
\hline Parameter (units) & $\begin{array}{l}\text { Spearman's correlation } \\
\text { coefficient }\left(\mathrm{r}_{\mathrm{s}}\right)\end{array}$ & $P$-value \\
\hline $\begin{array}{l}\text { L. infantum specific antibody } \\
\text { levels (EU) }\end{array}$ & -0.391 & 0.002 \\
Blood parasitemia (parasites/ml) & -0.413 & 0.001 \\
$\begin{array}{l}\text { IFN- } \text { concentration after } \\
\text { ConA stimulation (pg/ml) }\end{array}$ & 0.311 & 0.016 \\
IL-10 concentration after LSA & 0.585 & $<0.0001$ \\
$\begin{array}{l}\text { stimulation }(\mathrm{pg} / \mathrm{ml}) \\
\text { IL-10 concentration after }\end{array}$ & -0.81 & 0.562 \\
ConA stimulation $(\mathrm{pg} / \mathrm{ml})$ & & \\
\hline
\end{tabular}

Abbreviations: LSA L. infantum soluble antigen, ConA concavalin A

\section{Discussion}

In the present study, we demonstrated, for the first time, that dogs with several clinical stages of leishmaniosis presented differences in L. infantum specific cytokine profiles in stimulated blood. Interestingly, more than half of the dogs with clinical leishmaniosis showed a marked $L$. infantum specific IFN- $\gamma$ production. Canine clinical leishmaniosis is classically characterized by a reduced or absent L. infantum specific T-cell mediated immunity [25]. In contrast, Leishmania-specific IFN- $\gamma$ production in stimulated blood in dogs [26, 27] and other mammals such as rodents and humans have been associated with a resistant phenotype or with animals able to control infection [9]. However, more recent studies have already highlighted that T-cell mediated immunity might also be observed in dogs with clinical illness $[28,29]$ in agreement with the present results.

It is also important to remark that the majority of dogs (58 \%) that were L. infantum specific IFN- $\gamma$ producers were also classified with the lower clinical stages and were mainly classified as having stage I or stage IIa [6]. Furthermore, significant higher L. infantum IFN- $\gamma$ concentration was noted between stage I and IIa group and stage IIb, III and IV group. In addition, IFN- $\gamma$ producer dogs presented lower antibody levels as well as lower blood parasitemia when compared with IFN- $\gamma$ nonproducers. Moreover, significant negative correlations were found between LSA IFN- $\gamma$ concentration and antibody and blood parasitemia. Similar findings were observed in Brazil where pro-inflammatory cytokines such as IFN- $\gamma$ and TNF- $\alpha$ were highly expressed in the spleen of naturally infected dogs with low parasitism and these cytokines were negatively correlated with the parasite burden in the spleen [29]. An experimental canine model also demonstrated that splenic parasite burdens correlated negatively with Leishmania-specific IFN- $\gamma$ and IL-2 levels, and negatively with leishmanin skin test reactivity [30].
In the present study, $42 \%$ of the dogs were IFN- $\gamma$ non-producers and these dogs were most likely to be classified in higher clinical stages (stage IIb, III and IV) and also had high specific positive antibody levels and blood parasitemia as previously described in other studies in dogs with severe clinical leishmaniosis [19, 31]. It has been previously demonstrated that as dogs progress to clinical leishmaniosis, they display impaired CD4+ T cell proliferation and IFN- $\gamma$ production ex vivo in response to L. infantum antigen [19, 31]. However, the cellular basis and mechanisms for the development of antigen specific T-cell unresponsiveness in canine leishmaniosis are not fully understood. $\mathrm{T}$ cell exhaustion is well documented in chronic infections such as parasitic infections in several mammals. $\mathrm{T}$ cell exhaustion is defined as antigen-specific effector $\mathrm{T}$ cell dysfunction with sustained expression of inhibitory receptors, including programmed cell death protein 1 (PD-1) and decreased effector cytokine production such as IFN- $\gamma$ $[32,33]$. Interestingly, $T$ cell exhaustion was significantly associated with a four-fold increase in the population of $\mathrm{T}$ cells (both $\mathrm{CD}^{+}$and $\mathrm{CD}^{+} \mathrm{T}$ cells) with $\mathrm{PD}-1$ in a study comparing healthy non-infected control and sick foxhounds dogs with clinical leishmaniosis [31]. This was in conjunction with an absent lymphocyte proliferation and decreased peripheral blood mononuclear cells ( PBMC) L. infantum specific IFN- $\gamma$ production. These authors also demonstrated that PD-1 mediated exhaustion influenced macrophage-reactive oxygen intermediate production enabling the control of Leishmania infection [31]. We believe that T-cell exhaustion is probably the mechanism that explains $L$. infantum antigen Tcell unresponsiveness in our patients with moderate to very severe disease. Further studies should elucidate if PD-1 is diversely expressed in dogs in different clinical stages as the ones described in the present study.

Furthermore, in this study we have demonstrated that there were significant differences in IFN- $\gamma$ production after stimulation with ConA among clinical stages as well as between IFN- $\gamma$ producer and non-producer dogs. In stage IV-very severe disease, the IFN- $\gamma$ concentration after stimulation with ConA was significantly lower than in other less severe clinical stages (I, IIa and III) and the same tendency was noted with dogs in stage IIb. In addition, IFN- $\gamma$ non-producer dogs showed significantly lower IFN- $\gamma$ concentration after stimulation with ConA when compared with IFN- $\gamma$ producer dogs. These findings demonstrate that dogs with very severe disease are in more $\mathrm{T}$ cell anergy than dogs with less severe clinical stages. This was previously demonstrated by means of mitogenic lymphocyte proliferation such as conA in dogs with clinical leishmaniosis [34, 35].

Interestingly, our findings of IL-10 are not in agreement with the majority of studies on human visceral 
leishmaniosis $[36,37]$ or other studies reported in dogs with clinical leishmaniosis where a low or absent IFN- $\gamma$ production was found in tandem with increased production of IL-10 [19, 29]. An experimental canine model also demonstrated that splenic parasite burdens correlated positively with Leishmania-specific IL-10 levels [30]. Interestingly, a recent study carried out in Ethiopia in humans with visceral leishmaniosis due to $L$. donovani showed that Leishmania stimulated whole blood cells produce low or levels below the detection limit of IFN- $\gamma$ and IL-10 [38] in agreement with the results of IFN- $\gamma$ non-producer dogs of this study. In the present study, IFN- $\gamma$ producer dogs secreted higher levels of IL-10 concentration after LSA stimulation when compared with IFN- $\gamma$ non-producers. The reasons for these findings are difficult to explain but it could be because after LSA stimulation, IFN- $\gamma$ non-producer dogs might be less responsive to other cytokines including IL-10. Another possibility could be that IL-10 is produced in IFN- $\gamma$ producer dogs as a negative feedback to control proinflammatory cytokines as described in human mucocutaneous leishmaniosis [39]. IL-10 can counterbalance the proinflammatory effect of IFN- $\gamma$, leading to an adequate regulation of immune responses. In addition, it is important to remark that a high proportion of dogs did not produce IL10 and that, in general, IL-10 concentration was extremely much lower than IFN- $\gamma$ concentration. Moreover, IL-10 concentration after LSA stimulation was not associated with disease severity. Therefore, IL-10 does not appear to be a marker of disease severity.

Suppressed cell-mediated immunity in clinical canine leishmaniosis as a result of the inability of PBMCs to respond to Leishmania antigen, is thought to underlie the progressive nature of this disease [9]. In the present study, we reported the ability of a whole blood IFN- $\gamma$ release assay to detect dogs with clinical leishmaniosis with moderate to severe disease as previously reported in humans with visceral leishmaniosis due to $L$. donovani [40-43]. To date, there are few and poorly standardized assays to evaluate $L$. infantum specific T-cell mediated immunity responses in dogs [13] such as the leishmanin skin test $[14,15]$, lymphocyte proliferation assays $[16$, 17] and cytokine profiles in stimulated PBMC or blood from dogs $[44,45]$. This type of $L$. infantum specific whole blood assay has been described before in Leishmania vaccinated dogs $[44,45]$. However, to the best knowledge of the authors, this is the first time, that this whole blood assay has been performed in dogs with clinical leishmaniosis at different clinical stages. In human visceral leishmaniosis, whole blood assay that uses soluble Leishmania antigen have shown advantages over the leishmanin skin test, in terms of higher specificity and better correlation with surrogate markers of exposures to L. donovani [43]. Furthermore, in humans,
$\mathrm{CD} 4^{+} \mathrm{T}$ cells were found to be crucial for and the main source of the IFN- $\gamma$ production in Leishmania stimulated whole blood cultures [42] and we postulated that the same occurs in canines. We believe as described in human visceral leishmaniosis [43], that this assay is a promising tool to evaluate cellular immune responses in dogs in endemic areas. Research in this area is essential for the development of potential immunological and epidemiological tools for both canine and human leishmaniosis.

Our findings confirmed that canine leishmaniosis manifests in a wide spectrum of clinical illness as well documented elsewhere in clinical cases [46], studies [47] and guidelines $[6,48]$. The majority of dogs $(60 \%)$ were classified as LeishVet stage II (moderate disease) followed by stages I (16 \%), III (16 \%) and IV (6.6 \%). In addition, stage IIa (45\%) was the most frequently diagnosed clinical stage in the present study. The present results gave a good picture of the current presentation of clinical canine leishmaniosis at least in the north-east of Spain in client-owned dogs based on clinical stages. Our findings are likely to be similar to those present in other Mediterranean countries such as Portugal, France or Italy. It is important to highlight that the majority of the dogs studied did not have evidence of renal disease and, therefore, they had a good prognosis if managed with anti-Leishmania treatment.

\section{Conclusions}

The results of this study demonstrate that sick dogs lacking $L$. infantum specific IFN- $\gamma$ production in stimulated whole blood produce a strong humoral response, have a high blood parasitemia and severe clinical disease. In contrast, IL-10 does not appear to be a marker of disease severity. Pinpointing the precise immune responses associated with canine leishmaniosis will help to advance treatment and the development of a preventative strategy.

\section{Abbreviations \\ ALT, alanine aminotransferase; CBC, complete blood cell count; CD4, cluster of differentiation 4; ConA, concanavalin A; DNA, deoxyribonucleic acid; ELISA, enzyme-linked immunosorbent assay; EU, ELISA units; IFN- $\gamma$, interferon-gamma; IL-10, interleukin-10; IL-2, interleukin-2; IL-4, interleukin-4; IL-5, interleukin-5; LSA, L. infantum soluble antigen; NO, nitric oxide; PBMC, peripheral blood mono- nuclear cells; PD-1, programmed cell death protein 1; RPMI-1640, Roswell Park Memorial Institute 1640 medium; rt-PCR, real time PCR; TGF $\beta$, transforming growth factor beta; Th1, type 1 T helper lymphocytes; Th2, type 2 T helper lym- phocytes; TNFa, Tumor Necrosis Factor-alpha; UPC, urinary protein/creatinine ratio.}

\section{Acknowledgements}

The authors thank all veterinarians and dog owners that contributed to this study. Specially, we are grateful to Dr. Marta Planellas (Hospital Clínic Veterinari of Universitat Autonoma de Barcelona, UAB), Marta Blanchart (Ars Veterinaria), Montsant Osso (Consultori Falset), Annabel Dalmau (Hospital Mediterrani) and Joan Llull (Mon Veterinari). The authors are also grateful to Dra. Lorena Alborch, Daniel Marí, Paulina Quirola and Laura di Filippo (UAB) for the assistance with laboratory work. The authors also thank Dr. Shazia Hosein for English revision of the manuscript. 


\section{Funding}

This study was supported by a Spanish ministry grant, Ministerio de Economía y competitividad (AGL2012-32498). Publication fees of this manuscript have been sponsored by Bayer HealthCare - Animal Health division (Germany) in the framework of the 11th CVBD World Forum Symposium. Dr. Laia Solano-Gallego holds a Ramón y Cajal senior researcher contract awarded by the Ministerio de Ciencia e Innovación (Spain) and the European Social Fund.

\section{Authors' contributions}

LSG designed the research study. LSG supervised technical work. LSG and PMO contributed with data analysis and interpretation and wrote the manuscript. LO, LSG and PMO coordinated the veterinary clinics enrolled. PMO, SM and LO performed whole blood assay and collection of supernatants. PMO performed all serological and cytokine testing. SM performed all molecular work of this study. All authors read and approved the final version of the manuscript.

\section{Competing interests}

The authors declare that they have no competing interests.

\section{Ethics approval and consent to participate}

A signed informed consent was obtained from all owners. Residual samples of blood were used in this study, therefore, ethical approval was not required.

\section{Consent for publication}

Not applicable.

\section{Author details}

'Departament de Medicina i Cirurgia Animals, Facultat de Veterinària, Universitat Autònoma de Barcelona, Bellaterra, Spain. ${ }^{2}$ Hospital Clínic Veterinari, Universitat Autònoma de Barcelona, Bellaterra, Spain.

\section{Received: 14 March 2016 Accepted: 18 May 2016}

\section{Published online: 03 June 2016}

\section{References}

1. Hajjaran H, Mohebali M, Mamishi S, Vasigheh F, Oshaghi MA, et al. Molecular identification and polymorphism determination of cutaneous and visceral leishmaniasis agents isolated from human and animal hosts in Iran. BioMed Res Inter. 2013;2013:789326.

2. Baneth G, Zivotofsky D, Nachum-Biala Y, Yasur-Landau D, Botero AM. Mucocutaneous Leishmania tropica infection in a dog from a human cutaneous leishmaniasis focus. Parasit Vectors. 2014;7:118

3. Bamorovat M, Sharifi I, Dabiri S, Mohammadi MA, Fasihi Harandi M, et al. Leishmania tropica in stray dogs in Southeast Iran. Iran J Public Health. 2015:44:1359-66.

4. Carvalho FS, Wenceslau AA, Albuquerque GR, Munhoz AD, Gross E, Carneiro $\mathrm{PL}$, et al. Leishmania (Viannia) braziliensis in dogs in Brazil: epidemiology, co-infection, and clinical aspects. Genet Mol Biol. 2015;14:12062-73.

5. Baneth G, Koutinas AF, Solano-Gallego L, Bourdeau P, Ferrer L. Canine leishmaniosis - new concepts and insights on an expanding zoonosis: part one. Trends Parasitol. 2008;24:324-30.

6. Solano-Gallego L, Koutinas A, Miro G, Cardoso L, Pennisi MG, et al. Directions for the diagnosis, clinical staging, treatment and prevention of canine leishmaniosis. Vet Parasitol. 2009;165:1-18

7. Coffman RL, Seymour BW, Lebman DA, Hiraki DD, Christiansen JA, et al. The role of helper T cell products in mouse B cell differentiation and isotype regulation. Immunol Rev. 1988;102:5-28.

8. Mosmann TR, Cherwinski H, Bond MW, Giedlin MA, Coffman RL. Two types of murine helper T cell clone. I. Definition according to profiles of lymphokine activities and secreted proteins. 1986. J Immunol. 2005;175:5-14.

9. Hosein S, Blake D, Solano-Gallego L. Insights on adaptive and innate immunity in canine leishmaniosis. Parasitology. 2016;20:1-21.

10. Michel G, Pomares C, Ferrua B, Marty P. Importance of worldwide asymptomatic carriers of Leishmania infantum (L. chagasi) in human. Acta Trop. 2011;119:69-75.

11. Holzmuller P, Bras-Goncalves R, Lemesre JL. Phenotypical characteristics, biochemical pathways, molecular targets and putative role of nitric oxide-mediated programmed cell death in Leishmania. Parasitology. 2006;132(Suppl):S19-32.
12. Reis AB, Giunchetti RC, Carrillo E, Martins-Filho OA, Moreno J. Immunity to Leishmania and the rational search for vaccines against canine leishmaniasis. Trends Parasitol. 2010;26:341-9.

13. Fernandez-Bellon H, Solano-Gallego L, Rodriguez A, Rutten VP, Hoek A, et al. Comparison of three assays for the evaluation of specific cellular immunity to Leishmania infantum in dogs. Vet Immunol Immunopathol. 2005;107:163-9.

14. Solano-Gallego L, Llull J, Arboix M, Ferrer L, Alberola J. Evaluation of the efficacy of two leishmanins in asymptomatic dogs. Vet Parasitol. 2001;102:163-6.

15. Cardoso L, Neto F, Sousa JC, Rodrigues M, Cabral M. Use of a leishmanin skin test in the detection of canine Leishmania-specific cellular immunity. Vet Parasitol. 1998;79(3):213-20.

16. Rodriguez-Cortes A, Ojeda A, Francino O, Lopez-Fuertes L, Timon M, et al. Leishmania infection: laboratory diagnosing in the absence of a "gold standard". Am J Trop Med Hyg. 2010;82:251-6.

17. Strauss-Ayali D, Baneth G, Shor S, Okano F, Jaffe CL. Interleukin-12 augments a Th1-type immune response manifested as lymphocyte proliferation and interferon gamma production in Leishmania infantum-infected dogs. Int J Parasitol. 2005;35:63-73.

18. Moreno J, Vouldoukis I, Martin V, McGahie D, Cuisinier AM, et al. Use of a LiESP/QA-21 vaccine (CaniLeish) stimulates an appropriate Th1-dominated cell-mediated immune response in dogs. PLoS Negl Trop Dis. 2012;6:e1683.

19. Boggiatto PM, Ramer-Tait AE, Metz K, Kramer EE, Gibson-Corley K, et al. Immunologic indicators of clinical progression during canine Leishmania infantum infection. Clin Vaccine Immunol. 2010:17:267-73.

20. Solano-Gallego L, Villanueva-Saz S, Carbonell M, Trotta M, Furlanello T, et al. Serological diagnosis of canine leishmaniosis: comparison of three commercial ELISA tests (Leiscan, ID Screen and Leishmania 96), a rapid test (Speed Leish K) and an in-house IFAT. Parasit Vectors. 2014;7:111.

21. Solano-Gallego L, Alborch L, Di Filippo L, Planellas M, Montserrat S, et al. Rapid reduction of Leishmania infantum-specific antibodies during treatment in dogs with moderate disease. J Vet Inter Med. 2016;30:348-439.

22. Solano-Gallego, Ordeix L, Planellas M, Roura X, Altet L, et al. Early reduction of Leishmania infantum-specific antibodies and blood parasitemia during treatment in dogs with moderate or severe disease. Parasit Vectors. 2016;9:235.

23. Esteve LO, Saz SV, Hosein S, Solano-Gallego L. Histopathological findings and detection of Toll-like receptor 2 in cutaneous lesions of canine leishmaniosis. Vet Parasitol. 2015;209:157-63.

24. Martinez V, Quilez J, Sanchez A, Roura X, Francino O, et al. Canine leishmaniasis: the key points for qPCR result interpretation. Parasit Vectors. 2011;4:57.

25. Pinelli E, Killick-Kendrick R, Wagenaar J, Bernadina W, del Real G, et al. Cellular and humoral immune responses in dogs experimentally and naturally infected with Leishmania infantum. Infec Immun. 1994;62:229-35.

26. Pinelli E, van der Kaaij SY, Slappendel R, Fragio C, Ruitenberg EJ, et al. Detection of canine cytokine gene expression by reverse transcriptionpolymerase chain reaction. Vet Immunol Immunopathol. 1999;69:121-6

27. Santos-Gomes GM, Rosa R, Leandro C, Cortes S, Romao P, et al. Cytokine expression during the outcome of canine experimental infection by Leishmania infantum. Vet Immunol Immunopathol. 2002:88:21-30.

28. de Almeida Leal GG, Roatt BM, de Oliveira Aguiar-Soares RD, Carneiro CM, Giunchetti RC, et al. Immunological profile of resistance and susceptibility in naturally infected dogs by Leishmania infantum. Vet Parasitol. 2014:205:472-82.

29. do Nascimento PR, Martins DR, Monteiro GR, Queiroz PV, Freire-Neto FP, et al. Association of pro-inflammatory cytokines and iron regulatory protein 2 (IRP2) with Leishmania burden in canine visceral leishmaniasis. PLoS One. 2013:8:e73873

30. Aslan H, Oliveira F, Meneses C, Castrovinci P, Gomes R, et al. New insights into the transmissibility of Leishmania infantum from dogs to sand flies: experimental vector-transmission reveals persistent parasite depots at bite sites. J Infec Dis. 2016;213:1752-61.

31. Esch KJ, Juelsgaard R, Martinez PA, Jones DE, Petersen CA. Programmed death 1-mediated T cell exhaustion during visceral leishmaniasis impairs phagocyte function. J Immunol. 2013;191:5542-50.

32. Rodrigues V, Cordeiro-da-Silva A, Laforge M, Ouaissi A, Akharid K, et al. Impairment of T cell function in parasitic infections. PLoS Negl Trop Dis. 2014;8:e2567. 
33. Gigley JP, Bhadra R, Moretto MM, Khan IA. T cell exhaustion in protozoan disease. Trends Parasitol. 2012;28:377-84.

34. De Luna R, Vuotto ML, lelpo MT, Ambrosio R, Piantedosi D, et al. Early suppression of lymphoproliferative response in dogs with natural infection by Leishmania infantum. Vet Immunol Immunopathol. 1999;70:95-103.

35. Martinez-Moreno A, Moreno T, Martinez-Moreno FJ, Acosta I, Hernandez S. Humoral and cell-mediated immunity in natural and experimental canine leishmaniasis. Vet Immunol Immunopathol. 1995;48:209-20.

36. Kumar R, Nylen S. Immunobiology of visceral leishmaniasis. Front Immunol. 2012;3:251.

37. Gama ME, Gomes CM, Silveira FT, Laurenti MD, Goncalves Eda G, et al. Severe visceral leishmaniasis in children: the relationship between cytokine patterns and clinical features. Rev Soc Bras Med Trop. 2013;46:741-5.

38. Adem E, Tajebe F, Getahun M, Kiflie A, Diro E, et al. Successful treatment of human visceral leishmaniasis restores antigen-specific IFN-gamma, but not IL10 production. PLoS Negl Trop Dis. 2016;10:e0004468.

39. Nogueira RS, Gomes-Silva A, Bittar RC, Silva Mendonca D, Amato VS, et al. Antigen-triggered interferon-gamma and interleukin-10 pattern in cured mucosal leishmaniasis patients is shaped during the active phase of disease. Clin Exp Immunol. 2014;177:679-86.

40. Singh OP, Gidwani K, Kumar R, Nylen S, Jones SL, et al. Reassessment of immune correlates in human visceral leishmaniasis as defined by cytokine release in whole blood. Clin Vacc Immunol. 2012;19:961-6.

41. Gidwani K, Jones S, Kumar R, Boelaert M, Sundar S. Interferon-gamma release assay (modified QuantiFERON) as a potential marker of infection for Leishmania donovani, a proof of concept study. PLoS Negl Trop Diseas. 2011;5:e1042.

42. Kumar R, Singh N, Gautam S, Singh OP, Gidwani K, et al. Leishmania specific CD4 T cells release IFNgamma that limits parasite replication in patients with visceral leishmaniasis. PLoS Negl Trop Dis. 2014;8:e3198.

43. Singh OP, Sundar S. Whole blood assay and visceral leishmaniasis: challenges and promises. Immunobiology. 2014;219:323-8.

44. Carrillo E, Moreno J. Cytokine profiles in canine visceral leishmaniasis. Vet Immunol Immunopathol. 2009;28:67-70.

45. Carson C, Antoniou M, Ruiz-Arguello MB, Alcami A, Christodoulou V, et al. A prime/boost DNA/Modified vaccinia virus Ankara vaccine expressing recombinant Leishmania DNA encoding TRYP is safe and immunogenic in outbred dogs, the reservoir of zoonotic visceral leishmaniasis. Vaccine. 2009;27:1080-6.

46. Ordeix L, Solano-Gallego L, Fondevila D, Ferrer L, Fondati A. Papular dermatitis due to Leishmania spp. infection in dogs with parasite-specific cellular immune responses. Vet Dermatol. 2005;16:187-91.

47. Lombardo G, Pennisi MG, Lupo T, Chicharro C, Solano-Gallego L. Papular dermatitis due to Leishmania infantum infection in seventeen dogs: diagnostic features, extent of the infection and treatment outcome. Parasit Vectors. 2014;7:120.

48. Paltrinieri S, Solano-Gallego L, Fondati A, Lubas G, Gradoni L, et al. Guidelines for diagnosis and clinical classification of leishmaniasis in dogs. J Am Vet Med Assoc. 2010:236:1184-91.

\section{Submit your next manuscript to BioMed Central and we will help you at every step:}

- We accept pre-submission inquiries

- Our selector tool helps you to find the most relevant journal

- We provide round the clock customer support

- Convenient online submission

- Thorough peer review

- Inclusion in PubMed and all major indexing services

- Maximum visibility for your research

Submit your manuscript at www.biomedcentral.com/submit

) Biomed Central 